
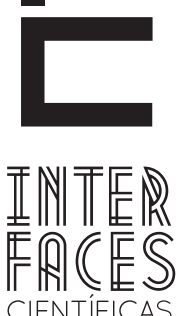

CIENTÍFICAS

SAÚDE E AMBIENTE

\title{
PERFIL SOCIODEMOGRÁFICO E EPIDEMIOLÓGICO DAS VÍTIMAS DE VIOLÊNCIA SEXUAL NO ESTADO DE SERGIPE
}

Laís Correia de Sousa ${ }^{1}$

Ana Carolina de Paula T. Miranda ${ }^{3}$
Leane de Carvalho Machado²

\section{RESUMO}

Dentre as manifestações de violência, a sexual é tida como a mais persistente e cruel, pois gera implicações traumáticas e permanentes para quem sofre. Atravessa décadas, permeia várias culturas, populações de diferentes faixas etárias e diversos espaços sociais, sendo, portanto, considerada como problema de saúde pública e de violação aos direitos humanos. 0 estudo objetivou caracterizar o perfil sociodemográfico e epidemiológico das vítimas de violência sexual notificadas no Serviço de Atendimento Médico a Vítimas de Violência Sexual. Estudo descritivo, de corte transversal, com coleta retrospectiva a partir dos prontuários das vítimas do serviço, no estado de Sergipe, no período de 2007 a 2011. A faixa etária das vítimas concentrou $56 \%$ de 10 a 14 anos e $94,3 \%$ do sexo feminino. Predominou a violência praticada por agressores conhecidos $(84,8 \%)$ e o IML encaminhou

$82,3 \%$ dos casos; $64 \%$ não fizeram uso de álcool e/ou drogas, enquanto $25 \%$ dos agressores fizeram uso. A procura por atendimento apontou $55,4 \%$ após $72 \mathrm{~h}$ da agressão e $57,7 \%$ apresentou ausência de lesões. A gravidez ocorreu em $6,3 \%$ dos casos e predominou a infecção por sífilis. A partir do conhecimento do perfil das vítimas de violência, espera-se que o presente estudo possa contribuir sensibilizando gestores e profissionais de saúde para o aprimoramento da atenção integral e humanizada ofertada por todos os serviços no atendimento identificando as vítimas de violência sexual.

\section{PALAVRAS CHAVE}

Violência Sexual. Vigilância. Doença Sexualmente Transmissível. 


\section{ABSTRACT}

Among the manifestations of violence, sex is seen as the most persistent and cruel because it generates traumatic and permanent implications for sufferers. Crosses decades, permeates many cultures, people of different ages and different social spaces, and is therefore considered as a public health problem and human rights violations. The study aimed to characterize the epidemiological and demographic profile of victims of sexual assault reported in the Medical Service for Victims of Sexual Violence. A descriptive, cross-sectional with retrospective from the medical records of victims of the service in the state of Sergipe, in the period 2007-2011. The age range of the victims concentrated $56 \% 10-14$ years and $94.3 \%$ were female. Predominated violence by aggressors known (84.8\%) and $82.3 \%$ referred IML cases, $64 \%$ did not use alcohol and / or drugs, while $25 \%$ of the aggressors had used. The demand for care showed $55.4 \%$ after $72 \mathrm{~h}$ of aggression and $57.7 \%$ had no lesions. Pregnancy occurred in $6.3 \%$ of cases and predominated syphilis infection. From the knowledge of the profile of the victims of violence, it is expected that this study will contribute sensitizing managers and health professionals to improve the integral and humanized services offered by all in attendance identifying victi$\mathrm{ms}$ of sexual violence.

\section{KEYWORDS}

Sexual Violence. Vigilance. Sexually Transmitted Disease.

\section{RESUMEN}

Entre las manifestaciones de la violencia, la agresión sexual es vista como la más persistente y cruel, ya que genera consecuencias traumáticas y permanentes para las víctimas. Cruza décadas, impregna muchas culturas, personas de diferentes edades y distintos espacios sociales, y por lo tanto se considera como un problema de salud pública y de violación de los derechos humanos. El estudio tuvo como objetivo caracterizar el perfil epidemiológico y sociodemográfico de las víctimas de asalto sexual reportado en el Servicio Médico para las Víctimas de la Violencia Sexual. Estudio descriptivo, transversal, con recogida retrospectiva de las historias clínicas de las víctimas del servicio en el estado de Sergipe, en el período 2007-2011. La franja etaria de las víctimas concentra un $56 \%$ entre los 10 y los 14 años y un $94,3 \%$ de mujeres. Predominó la violencia practicada por autores conocidos $(84,8 \%$ ) y el IML envió un $82,3 \%$ de los casos; $64 \%$ no utilizaron alcohol y / o drogas, mientras que el $25 \%$ de los autores había utilizado. La busca por asistencia fue de $55,4 \%$ después de 72 horas de la agresión y el $57,7 \%$ no presentaba lesiones. El embarazo ocurrió en el 6,3\% de los casos y la infección por sífilis predominó. A partir del conocimiento del perfil de las víctimas de violencia, se espera que este estudio contribuya para la sensibilización de autoridades y profesionales de la salud para mejorar la atención integral y humanizada ofrecida por todos los establecimientos que ofrecen asistencia e identificación a las víctimas de violencia sexual.

\section{Palabras clave:}

Violencia Sexual. Vigilancia. Enfermedad de Transmisión Sexual 


\section{INTRODUÇ̃̃̃O}

A violência representa um dos mais graves problemas de saúde no mundo e o seu impacto não representa um fenômeno localizado, está presente em vários países, desenvolvido ou não, caracterizando-se como epidemia e, portanto um problema de saúde pública (HENRIQUES, 2004).

Inicialmente a violência estava restrita à área da segurança pública e do direito criminal. Apenas na década de 1960, a pediatria americana se aprofundou no tema, a fim de estudar a síndrome do bebê espancado, já que interferia diretamente no seu crescimento e desenvolvimento. Nos anos 1970, programas de prevenção primária e secundárias e locais para denúncia foram criados, tornando público um problema até então considerado de foro privado. No Brasil, somente a partir da década de 1980 a violência começa a ser debatida e entra no setor saúde se firmando na década de 1990 (MINAYO; SOUZA, 1999).

O Relatório Mundial sobre Violência e Saúde, define violência como:

Uso intencional de força física ou do poder, real ou em ameaça, contra si próprio, contra outra pessoa, contra um grupo ou uma comunidade que resulte ou tenha possibilidade de resultar em lesão, morte, dano psicológico, deficiência de desenvolvimento ou privação (KRUG et al., 2002, p. 5).

Os comportamentos humanos violentos são fenômenos complexos e provoca grande impacto no perfil da morbi-mortalidade, fato esse que reflete no aumento das demandas aos serviços de saúde, sobrecarregando não somente o atendimento ao trauma, mas também aqueles relacionados à recuperação, reabilitação física e atendimento psicológico (HENRIQUES, 2004).

Dentre todas as manifestações de violência, a sexual é tida como a mais persistente e cruel, pois gera implicações traumáticas e permanentes para quem sofre. Possui proporções de uma pandemia, pois atravessa décadas, permeia por várias culturas, populações de diferentes faixas etárias e diversos espaços sociais, principalmente o doméstico. Por vezes, produz vulnerabilidades e promove sensações de insegurança, colaborando para manutenção de uma cultura patriarcal e violenta, sendo considerada, portanto, como problema de saúde pública e de violação aos direitos humanos (BRASIL, 2012).

\section{A violência sexual é considerada como,}

Revela-se de várias formas, tais como assédio sexual, estupro, jogos sexuais, pornografia infantil, sexo forçado no casamento, voyeurismo, práticas eróticas impostas, etc. 0 assédio sexual consiste em constranger o individuo, com propostas, perguntas ou qualquer forma de abordagem forçada de natureza sexual. A pornografia infantil é caracterizada pela divulgação em qualquer meio de comunicação de imagens com pornografia ou cenas de sexo explícito envolvendo crianças ou adolescentes (BRASIL, 2011).

Já a exploração sexual ocorre quando há utilização de pessoas com intenções de comercialização e obtenção de lucro, seja para a prática de relações sexuais, seja para a exposição da nudez ou publicações de imagens em meios de comunicação. E o estupro se dá quando o indivíduo obriga, mediante violência ou ameaça, a ter relações sexuais, que pode incluir penetração peniana ou qualquer objeto na vagina, ânus ou boca, independente da orientação sexual ou do sexo da vítima (BRASIL, 2011). 
A violência sexual não somente gera consequências para a saúde física, pois existe o risco de contaminação por Doenças Sexualmente Transmissíveis (DST's), e até mesmo o risco da gravidez indesejada, tornando pior o estado traumático. Estas consequências podem ser agravadas, gerando síndrome do pânico, depressão, distúrbios psicossomáticos e ansiedade (BRASIL, 2012).

As condutas clínicas na assistência à vítima de violência sexual se baseiam no tempo em que a vítima procura o serviço, que deve ser, preferencialmente, iniciado dentro das primeiras 72 horas após a violência, considerando a maior eficácia de algumas intervenções nesse período. Sendo assim, o atendimento ao caso de violência sexual deve criar rotinas para garantir de imediato a realização do exame físico de forma completa, coleta de amostras para diagnóstico de infecções genitais, exame ginecológico, coleta de material para identificação do provável autor(a) da agressão, além disso, assegurar o preenchimento da Ficha de Notificação e Investigação de Violência Doméstica, Sexual e/ou outras Violências (BRASIL, 2012).

Nos casos de ocorrência de gravidez, seja ela suspeita ou confirmada, cabe aos profissionais de saúde fornecer informações sobre os direitos das mulheres e apresentar alternativas como a interrupção da gravidez, a assistência pré-natal e entrega da criança para adoção, respeitando sempre a autonomia da mulher, verificando se existe desejo ou não de interromper a gravidez (BRASIL, 2012).

\section{METODOLOGIA}

Trata-se de um estudo descritivo, de corte transversal, com coleta retrospectiva e abordagem quantitativa realizado no Serviço de Atendimento Médico às Vítimas de Violência Sexual no período de 2007 a
Os reflexos da violência são claramente notados no âmbito dos serviços de saúde, seja pela complexidade do atendimento que demandam, seja pelos custos que representam. Dessa forma, o setor saúde tem importante papel no enfrentamento à violência (SALIBA et al., 2007).

Em 25 de janeiro de 2011, o Ministério da Saúde publicou a portaria GM/MS n ${ }^{0} 104$, onde acrescenta à lista de agravos de notificação compulsória qualquer suspeita ou confirmação de violência sexual cometida contra crianças, adolescentes, mulheres e pessoas idosas. Dessa forma, as notificações devem ser realizadas através da Ficha de Notificação e Investigação de Violência Doméstica, Sexual e/ou outras Violências, por todos os serviços de saúde, seja ele público ou privado, e não restrita às unidades sentinelas e órgãos públicos, como anteriormente (BRASIL, 2011).

A rotina de notificação e a divulgação dos dados sintetizados são poderosos instrumentos de vigilância e monitoramento, uma vez que ajudam a dimensionar a questão da violência e conhecer sua dinâmica, a determinar a necessidade de investimentos em núcleos de vigilância e assistência e permitir o planejamento das ações de prevenção e proteção, propiciando a elaboração de políticas públicas e a compreensão da eficácia de intervenções (MINAYO; SOUZA, 1999).

Levando-se em consideração a importância da temática, o estudo objetivou caracterizar o perfil sociodemográfico e epidemiológico das vítimas de violência sexual residentes no estado de Sergipe no período de 2007 a 2011.

2011. Este serviço funciona na Maternidade Nossa Senhora de Lourdes (MNSL), em Aracaju desde junho de 2004, oferecendo assistência médica, psicológica, assistência social e de enfermagem durante 24 horas. 
Os dados foram coletados através de prontuários de vítimas de estupro e a amostra foi calculada através da fórmula de Barbetta (MEDRONHO et al., 2004), levando-se em consideração o universo de 309 vítimas de estupro, residentes no estado de Sergipe, e atendidas na MNSL. Obteve-se como amostra 175 vítimas de violência sexual selecionados de forma aleatória simples.

As variáveis extraídas dos prontuários das vítimas atendidas na MNSL foram: faixa etária; sexo; vínculo com o agressor; modo de entrada; uso de álcool/ drogas pelo agressor e pela vítima; tempo decorrente entre a violência e o atendimento; tipos de lesão corporal; presença de gravidez decorrente da violência; decisão da mulher ou representante legal quanto ao prosseguimento da gravidez e Investigação de DST/ Hepatites/HIV.

\section{RESULTADOS}

O estudo realizado nos 175 prontuários das vítimas de estupro, atendidas na Maternidade Nossa Senhora de Lourdes, apontou vitima mais jovem com 02 anos e a de maior idade com 48 anos e a média foi de 14 anos; a faixa etária mais acometida localizou-se entre 10 e 14 anos totalizando $56 \%$ (98) dos casos. Em relação ao sexo, houve prevalência de 94,3\% (165) para o sexo feminino (Tabela 1).

Em relação ao vínculo que a vítima possuía com o agressor, $84,6 \%$ (148) informam que conheciam o agressor, no entanto, $49,1 \%$ (86) relataram o tipo de vinculo com o agressor e 35,4\% (62) não informaram o grau de intimidade; apenas 15,4\% (27) informaram agressor desconhecido. (Tabela 2).

Quanto ao modo de entrada das vítimas na MNSL, observou-se que dentre os estabelecimentos existen-
A tabulação e consolidação dos dados foram realizadas através do programa Excel 2007- Microsoft Office. A análise dos dados foi realizada de forma descritiva através de gráficos e tabelas, média, frequência absoluta e relativa.

O estudo obteve aprovação do Comitê de Ética e Pesquisa da Universidade Tiradentes para coleta de dados na SMS de Aracaju e na MNSL através do protocolo no 061112 atendendo a resolução 196/96 do Conselho Nacional de Saúde.

A pesquisa ofereceu riscos mínimos, visto que poderia trazer constrangimento, os quais foram minimizados com a preservação das informações coletadas, onde os usuários foram identificados apenas numericamente.

tes no Estado de Sergipe, o Instituto Médico Legal (IML) é o que mais encaminha as vítimas para a MNSL com 82,3\% (144) seguido de demanda espontânea com 13,7\% (24) e apenas 1,8\% (03) das Unidades Básicas de Saúde (UBS) dos municípios de Sergipe (Lagarto, Divina Pastora e Carmópolis) (Tabela 3).

No que se refere ao uso de álcool e/ou drogas pelo agressor no momento da violência, 64\% (101) das vítimas relataram que os agressores tinham feito uso de algum tipo de substância que altera o comportamento. Em relação ao uso de álcool e/ou drogas pela vítima, 46\% (72) afirmaram que utilizou algum tipo de substância. (Figura 1).

A variação entre a ocorrência da violência e o tempo de chegada ao serviço de referência mostrou variação de $\leq 24$ horas até 09 anos sendo que 55,4\% (97) procuraram o serviço após 72 horas (Tabela 4). 
Tabela 1 - Distribuição dos casos de estupro segundo faixa etária e sexo no período de 2007 a 2011 no estado de Sergipe, Brasil

\begin{tabular}{ccccccc}
\hline Sexo & \multicolumn{2}{c}{ Feminino } & \multicolumn{2}{c}{ Masculino } & \multicolumn{2}{c}{ Total } \\
\hline Idade & $\mathrm{n}$ & $\%$ & $\mathrm{~N}$ & $\%$ & $\mathrm{~N}$ & $\%$ \\
$\leq 4 \mathrm{a}$ & 7 & 4,2 & 1 & 10 & 8 & 4,6 \\
5 a $9 \mathrm{a}$ & 15 & 9,1 & 4 & 40 & 19 & 10,9 \\
10 a 14 a & 94 & 57,0 & 4 & 40 & 98 & 56,0 \\
15 a 19 a & 31 & 18,8 & 1 & 10 & 32 & 18,3 \\
20 a 29 a & 11 & 6,7 & 0 & 0 & 11 & 6,3 \\
30 a 29a & 4 & 2,4 & 0 & 0 & 4 & 2,3 \\
$>40 \mathrm{a}$ & 3 & 1,8 & 0 & 0 & 3 & 1,7 \\
Total & 165 & 100,0 & 10 & 100 & 175 & 100,0 \\
\hline
\end{tabular}

Fonte: Secretaria de Estado da Saúde de Sergipe

Tabela 2 - Distribuição dos casos de estupro segundo vínculo e tipo de vínculo com o agressor no período de 2007 a 2011 no estado de Sergipe, Brasil

\begin{tabular}{ccc}
\hline Vínculo & $\mathbf{N}$ & \% \\
\hline Conhecidos & 148 & 84,6 \\
Desconhecidos & 27 & 15,4 \\
Total & 175 & 100 \\
Tipo de vínculo & & \\
Padrasto & 20 & 11,4 \\
Namorado & 17 & 9,7 \\
Vizinho & 12 & 6,9 \\
Pai & 9 & 5,1 \\
Primo & 6 & 3,4 \\
Tio & 5 & 2,9 \\
Ignorado & 4 & 2,3 \\
Irmão & 4 & 2,3 \\
Cunhado & 3 & 1,7 \\
Amigo & 2 & 1,1 \\
Marido/Companheiro & 2 & 1,1 \\
Ex-namorado & 1 & 0,6 \\
Professor & 1 & 0,6 \\
Total & 86 & 49,1 \\
\hline
\end{tabular}

Fonte: Secretaria de Estado da Saúde de Sergipe 
Tabela 3 - Distribuição de casos de estupro, segundo modo de entrada no período de 2007 a 2011 no estado de Sergipe, Brasil

\begin{tabular}{ccc}
\hline Modo de entrada & N & \% \\
\hline Encaminhamento IML & 144 & 82,3 \\
Demanda espontânea & 24 & 13,7 \\
Encaminhamento SSP & 3 & 1,7 \\
Encaminhamento HUSE & 1 & 0,6 \\
Encaminhamento UBS & 3 & 1,8 \\
Total & 175 & 100,0 \\
\hline
\end{tabular}

Fonte: Secretaria de Estado da Saúde de Sergipe

Figura 1 - Distribuição de casos de estupro, segundo uso de álcool/drogas pelo autor e vítima da agressão no período de 2007 a 2011 no estado de Sergipe, Brasil

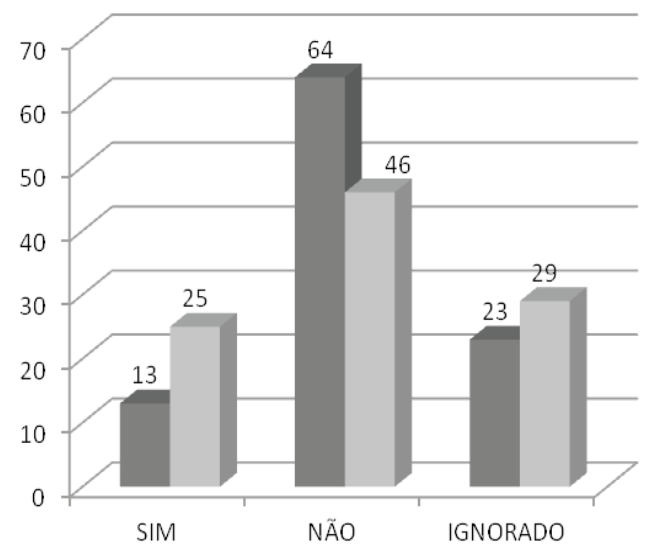

- Paciente

Autor da agressão

Fonte: Secretaria de Estado da Saúde de Sergipe

Tabela 4 - Distribuição de casos de estupro, segundo tempo decorrido desde a violência sexual até o atendimento médico no período de 2007 a 2011 no estado de Sergipe, Brasil

\begin{tabular}{|c|c|c|}
\hline Tempo decorrente entre a violência e $\mathrm{o}$ atendimento & $\mathbf{N}$ & $\%$ \\
\hline$\leq 24 h$ & 45 & 25,8 \\
\hline $25 \mathrm{a} 48 \mathrm{~h}$ & 19 & 5,1 \\
\hline $49 a 72 h$ & 5 & 2,9 \\
\hline$\geq 72 \mathrm{~h}$ a 09 anos & 97 & 55,4 \\
\hline Ignorado & 19 & 10,9 \\
\hline Total & 175 & 100,0 \\
\hline
\end{tabular}

Fonte: Secretaria de Estado da Saúde de Sergipe 
Dos 175 prontuários coletados, $57,7 \%$ (101) das vitimas apresentaram ausência de lesões físicas decorrentes da agressão e em $16(9,1 \%)$ casos, obtiveram traumas genitais. Destaca-se que em 26,3\% (46) prontuários não constavam informação acerca da presença de traumas, sendo classificados como ignorado (Tabela 5).

Tabela 5 - Distribuição de casos de estupro, segundo tipos de lesões corporais no período de 2007 a 2011 no estado de Sergipe, Brasil

\begin{tabular}{ccc}
\hline Traumas & N & \% \\
Genital & 16 & 9,1 \\
Extragenital & 09 & 5,1 \\
Genital e extragenital & 03 & 1,7 \\
Ausente & 101 & 57,7 \\
Ignorado & 46 & 26,3 \\
Total & 175 & 100 \\
\hline
\end{tabular}

Fonte: Secretaria de Estado da Saúde de Sergipe

0 estudo apontou $6,7 \%$ (11) das vítimas de estupro resultaram em gravidez. 0 resultado da decisão legal da mulher ou do seu representante legal mostrou $36,4 \%$ (04) das que engravidaram optaram pelo aborto legal, $27,3 \%$ (03) decidiram prosseguir com a gravidez realizando o pré-natal e $36,4 \%$ (04) não retornaram para as consultas para informar sobre decisão que tomaram. É importante ressaltar que 01 desses abortos foi de forma espontânea, segundo registros no prontuário (Tabela 6).

Tabela 6- Distribuição de casos de estupro, segundo ocorrência de gravidez e sua decisão no período de 2007 a 2011 no estado de Sergipe, Brasil

\begin{tabular}{ccc}
\hline Gravidez & $\mathbf{n}$ & $\mathbf{\%}$ \\
\hline Reagente & 11 & 6,3 \\
Não reagente & 164 & 93,7 \\
Total & 175 & 100,0 \\
Decisão da Mulher & & 36,4 \\
Aborto & 4 & 27,3 \\
Pré-Natal & 3 & 36,4 \\
Não Retornou & 4 & 100,0 \\
Total & 11 & \\
\hline
\end{tabular}

Fonte: Secretaria de Estado da Saúde de Sergipe

Das 175 vítimas de estupro, 10,9\% (19) teve resultado positivo para DST. Das principais DST's encontradas, 52,6\% (10) das vitimas tiveram infecção por clamídia (Tabela 7). 
Tabela 7 - Distribuição de casos de estupro, segundo DST diagnosticadas no período de 2007 a 2011 no estado de Sergipe, Brasil

\begin{tabular}{ccc}
\hline DST & $\mathbf{N}$ & $\mathbf{\%}$ \\
\hline Presente & 19 & 10,9 \\
Ausente & 156 & 89,1 \\
Total & 175 & 100,0 \\
Agravo & & \\
Clamídia & 10 & 52,6 \\
Sífilis & 3 & 15,8 \\
Hepatite C & 2 & 10,5 \\
Hepatite B & 1 & 5,3 \\
Trichomonas Vaginalis & 1 & 5,3 \\
HPV & 1 & 5,3 \\
Total & 19 & 100,0 \\
\hline
\end{tabular}

Fonte: Secretaria de Estado da Saúde de Sergipe

\section{DISCUSSÃO}

Estudo mostrou maior número de vítimas de estupro com idade entre 10 a 14 anos, sendo o sexo feminino predominante em todas as faixas etárias apontando que as mulheres mais jovens correm um risco maior de sofrer esse tipo de agressão. Em relação à faixa etária, os dados são concordantes com Andrade e outras (2001) onde estudo realizado com fichas de atendimentos de mulheres vítimas de violência sexual, em Curitiba, apontou maior ocorrência entre jovens menores de 20 anos.

Estudo evidenciou que a maioria das agressões é praticada por conhecidos das vítimas: aqueles que eram conhecidos e as vítimas relataram o tipo de vínculo, e aqueles classificados como conhecidos e as vítimas não referiram o grau de intimidade. Este fato pode ser explicado pelo provável medo que a vítima tem de expor a identidade do agressor. Pesquisa realizada em prontuários de crianças por Monteiro et al. (2008), em Teresina/ Piauí, evidenciou como principal agressor o vizinho. Já Ramos et al. (2009), pesquisou prontuários de mulheres vítimas de violência sexual, em Santo André/SP, e obser- vou predomínio das agressões perpetradas por desconhecidos 79,3\%, discordando do presente estudo.

O IML foi quem realizou o maior número de encaminhamento dos casos, provavelmente por ser esse o serviço que realiza o exame pericial das vítimas de estupro no estado de Sergipe. Observa-se uma baixa quantidade de encaminhamentos pelas UBS, apesar de ser o contato preferencial dos usuários e a principal porta de entrada ao sistema de saúde.

Essas unidades deveriam acolher os usuários promovendo a vinculação e corresponsabilização pela atenção às suas necessidades de saúde (BRASIL, 2012). 0 estudo evidencia a necessidade de sensibilizar e capacitar os profissionais das UBS para a importância e a necessidade de identificar sinais e sintomas de violência; fortalecer o vinculo com o usuário levando em consideração o receio de não ser acolhido, por conta do constrangimento vivido, gerando silencio perante o sofrimento; e realizando encaminhamento para o local adequado. 
0 uso de álcool e/ou drogas pelos agressores estava presente em $25 \%$ dos casos apontando situação mais grave em Sergipe quando comparado com estudo realizado por Lopes et al. (2004), em Teresina/Piauí que apontou $18,6 \%$ dos agressores tinham utilizado algum tipo de substância que provoque distúrbios. 0 uso de álcool e/ou drogas pelas vítimas no momento da violência foi de $13 \%$. Pesquisa feita por Collins \& Messerschimdt (1993), não considerou como fator de risco para a violência sexual, o uso de álcool e/ou drogas pelas vítimas. Estudo feito por Guerra (2000) relata que o estupro tem sido comumente associado ao abuso de drogas, já a violência doméstica está relacionada ao abuso de álcool.

A maioria das vítimas procurou o serviço após $72 \mathrm{~h}$ da ocorrência da agressão. Esses dados discordam da pesquisa realizada por Oliveira et al. (2006), com prontuários de vítimas de estupro, em Londrina/Pr, onde apenas $14,2 \%$ das vítimas procuraram atendimento após 72h. Quanto mais precoce a vítima procurar o serviço, maior será a eficácia da quimioprofilaxia para as DST's e da anticoncepção de emergência, estas devem ser feitas nas primeiras 72 horas, preferencialmente no primeiro dia do ato agressivo (BRASIL, 2012).

O elevado número de atraso na procura do atendimento, provavelmente se deva ao fato do desconhecimento da existência do serviço de referência para vítimas de violência sexual, falta de informação quanto aos riscos de adquirir DST's, vergonha de se expor e muitas vezes se sentir culpada pelo fato ocorrido.

Houve predomínio das vítimas que não apresentaram lesões decorrentes da violência. Pesquisa realizada por Lopes et al. (2004), em Teresina/Piauí, mostrou que $76,7 \%$ dos casos, houve lesão física decorrente da agressão sexual. A ausência de lesões genitais e extragenitais, detectadas no estado de Sergipe durante $o$ atendimento não determina a inexistência de violência sexual, já que muitas vezes, as marcas da violência física não são evidenciadas devido à demora na procura pelo serviço de saúde, onde provavelmente já estabeleceu os processos cicatriciais, não havendo mais presença de lesões evidentes de uma agressão sexual recente.

A quantidade de dados ignorados relacionados ao tipo de lesão sofrida demonstra provavelmente um desinteresse ou mesmo descuido durante o registro das informações, pelo profissional de saúde que realizou o atendimento, apontando a necessidade de realizar sensibilização para a problemática.

Quanto à ocorrência de gravidez, os dados não estão em consonância com pesquisa de Mattar et al. (2007) em São Paulo, onde descreveu que 30\% dos casos houve gestação e em $63 \%$ delas houve interrupção. Brasil (2012), afirma que a estimativa de gravidez decorrente da violência sexual varia entre 01 a $05 \%$, enquanto o presente estudo aponta índice mais elevado (6,3\%).

O risco de adquirir a infecção por sífilis e clamídia após um estupro foi alta nesta pesquisa quando comparado com o estudo realizado por Jenny et al. (1990), com vítimas de estupro, em Londres, observou que a estimativa de infecção por clamídia após a violência sexual varia entre 1,5 a $16 \%$, para sífilis a taxa é de $1 \%$. Já para trichomonas a taxa é de $14,7 \%$.

Provavelmente essa alta incidência ocorre devido à alta prevalência destas doenças na população em estudo, em comparação com os outros agentes patogênicos. Além disso, existe a impossibilidade de distinguir entre infecções pré-existentes e as que resultaram da presença de sêmen infectado do agressor.

A taxa de infecção de DST's não virais está entre 16 a $58 \%$, esta estimativa varia de acordo com o agente infeccioso (BRASIL, 2012). Segundo Crowe et al. (1996), a estimativa de transmissão da hepatite B nos casos decorrentes do contato sexual é desconhecido. No entanto, nos casos contaminação por via percutânea com sangue HbsAg positivo, a estimativa é de $30 \%$. Apesar da alta estimativa de infecção por 
Hepatite $B$, nesta pesquisa houve apenas 01 caso de contaminação.

As taxas de infecção por DST's estão relacionadas a ejaculação durante a agressão, assim como o número de agressores, a infectividade dos organismos transmitida, o tipo de penetração (vaginal, anal ou oral), a suscetibilidade da vítima ter infecção, a profilaxia adequada, a ocorrência de traumatismos genitais e a presença de DST ou úlcera genital prévia (BRASIL, 2012).

A pesquisa não encontrou vítimas que tenham sorologias positivas para HIV, o que mostra concordância com a literatura. Jenny et al. (1990), refere estimativa de infecção para HIV de 0,8\%.

\section{CONCLUSÃO}

O estudo evidenciou a importância do registro correto do atendimento nos prontuários das vítimas e a necessidade de realizar sensibilização dos profissionais de saúde, especialmente das UBS, para aprimorar a identificação dos casos de violência sexual, acolhendo, aconselhando, registrando adequadamente e encaminhando oportunamente para o serviço de referência.

A violência sexual favorece o aparecimento de outros agravos e o seu reconhecimento precisa ser tratado como problema de saúde pública; para tanto, cabe ao poder público, ações mínimas efetivas, promovendo maior investimento na segurança, integração entre os serviços de saúde, além da priorização de medidas preventivas que visem à redução da incidência de DST.
A quantidade de usuários que deixam de ser atendidos após a violência é grande e a procura pelo atendimento é feita tardiamente. Sugere-se realizar uma ampla divulgação na mídia falada, escrita e televisiva, sobre o Serviço de Atendimento à Vítima de Violência Sexual de Sergipe e a importância de procurar o mais rápido possível o atendimento.

Há necessidade de continuar realizando estudos sobre a temática e que também abordem as vítimas de violência sexual do sexo masculino, devido à escassez de dados referentes a esta categoria. Espera-se que este estudo sensibilize os gestores e profissionais de saúde para o aprimoramento da atenção integral e humanizada ofertada por todos os serviços no atendimento às vítimas de violência.

\section{REFERÊNCIAS}

ANDRADE, R.P; GUIMARÃES, A.C.P.;FILHO, A.F.; CARVALHO, N.S.; ARRABAL, J.S.; ROCHA, D.M.; MEDEIROS, J.M. Características Demográficas e Intervalo para Atendimento em Mulheres Vítimas de Violência Sexual. Revista Brasileira de Ginecologia e Obstetrícia, 2001.

BRASIL, Decreto No 5.099 de 3 de junho de 2004. Regulamenta a Lei nº 10.778, de 24 de novembro de 2003 , e institui os serviços de referência sentinela. Brasília, 2004.

BRASIL, Lei n 8.069 de 13 de julho de 1990. Estatuto da Criança e do Adolescente. Brasília, 1990. 
BRASIL. Ministério da Saúde - Prevenção e Tratamento dos Agravos Resultantes da Violência Sexual Contra Mulheres e Adolescentes. Norma Técnica. 3. ed. Série A. Normas e Manuais Técnicos, Série Direitos Sexuais e Direitos Reprodutivos - Caderno nº 6 Brasília - DF. 2012.

BRASIL. Ministério da Saúde. Portaria GM/MS nº 104, de 25 de janeiro de 2011. Define as terminologias adotadas em legislação nacional, conforme disposto no Regulamento Sanitário Internacional 2005, a relação de doenças, agravos e eventos em saúde pública de notificação compulsória em todo o território nacional e estabelece fluxo, critérios, responsabilidades e atribuições aos profissionais e serviços de saúde. Diário Oficial da União, Brasília, n. 18, Seção 1, p. 37-38, 26 de janeiro de 2011.

BRASIL. Ministério da Saúde. Secretaria de Vigilância em Saúde. Departamento de Análise de Situação de Saúde. Viva: instrutivo de notificação de violência doméstica, sexual e outras violências / Ministério da Saúde. Secretaria de Vigilância em Saúde. Departamento de Análise de Situação de Saúde. - Brasília: Ministério da Saúde, 2011.

COLLINS, J. \& MESSERSCHIMDT, P. Epidemiology of alcohol-related violence. Alcohol. Health and Research World, 1993.

CROWE, C.; FORSTER, G.E.; DINSMORE, W.W.; MAW, R.D. A case of acute hepatitis B ocurring four months after multiple rape. International Journal of STD \& AIDS, 1996.

GUERRA, C.C.; Ei tá violência conjugal e familiar nossa de cada dia. Dados levantados nos prontuários do S.0.S. Mulher/Família de Uberlândia. Gênero em Pesquisa, 2000.

HENRIQUES, C.V. Perfil Clínico-Epidemiológico das Mulheres Vítimas de Violência Atendidas no Serviço de Apoio à Mulher, Recife-PE. Instituto Materno-Infantil de Pernambuco (Imip). Recife, 2004.

JENNY, C. et al., Sexually transmitted diseases in rape victims. Genitourin Med, 1990.

KRUG, E.G. et al., Organização Mundial da Saúde. Relatório Mundial sobre Violência e Saúde. Geneva, 2002.

LOPES, I.M.R.S.; GOMES, K.R.O.; SILVA, B.B.; DEUS, M.C.B.R.; GALVÃO, E.R.C.G.N.; BORBA, D.C. Caracterização da Violência Sexual em Mulheres Atendidas no Projeto Maria-Maria em Teresina-PI. Revista Brasileira de Ginecologia e Obstetrícia, 2004.

MATTAR, R.; ABRAHÃO, A.R.; NETO, J.A.; COLAS, O.R.; SCHROEDER, I.; MACHADO, S.J.R.; MANCINI, S.; VIEIRA, B.A.; BERTOLANI, G.B.M. Assistência multiprofissional à vítima de violência sexual: a experiência da Universidade Federal de São Paulo. Cad. Saúde Pública, Rio de Janeiro, 2007.

MEDRONHO, R.A.; BLOCH, K.V.; LUÍZ, R.R.; WERNEK, G.L. Epidemiologia. 2. ed. Atheneu: São Paulo, 2004.

MINAYO, M.C.S; SOUZA, E.R. É possível prevenir a violência? Reflexões a partir do campo da saúde pública. Associação Brasileira de Pós-Graduação em Saúde Coletiva Brasil. Ciência e Saúde Coletiva, 1999. 
MONTEIRO, CFS, et al. Violência Sexual contra criança no meio intrafamiliar atendidas no SAMVVIS, em Teresina-PI. Revista Brasileira de Enfermagem, 2008.

OLIVEIRA, P.M.; CARVALHO, M.L.O. Perfil das mulheres atendidas no Programa Municipal de Atendimento à Mulher Vítima de Violência Sexual em Londrina-PR e as circunstâncias da violência sexual sofrida: período de outubro de 2001 a agosto de 2004. Semina: Ciências Biológicas e Saúde, Londrina, 2006.

ORGANIZAÇÂO MUNDIAL DE SAÚDE - Relatório mundial sobre violência e saúde. Genebra. 2002. Disponível em: <http://www.redesaude.org.br/portal/home/conteudo/biblioteca/biblioteca/textos-diversos/037.pdf>. Acesso em: 3 set. 2012.

RAMOS, C.R.A.; MEDICCI, V.P.G.; PUCCIA, M.I.R. Mulheres vitimadas sexualmente - perfil sócio demográfico e análise do atendimento em um centro de referência. Revista Inst. Ciências da Saúde, 2009.

SALIBA, O.; GARBIN, C.A.S.; GARBIN, A.J.I.; DOSSI, A.P. Responsabilidade do profissional de saúde sobre a notificação de casos de violência doméstica. Revista Saúde Pública, 2007. 